\title{
Fluence-dependent electron energy loss spectroscopy mapping for beam-sensitive polymers
}

\author{
Robert Colby and Donald Carpenter \\ EMRE, United States
}

Polymer blends and composites would benefit from high spatial resolution mapping with interpretable chemical contrast. Electron energy loss spectroscopy (EELS) can identify polymer type, and potentially even subtler changes to bonding, packing, and molecular orientation. However, most polymers evidence beam-induced changes at fluences well less than 10 electrons $/ \AA^{2}$. For a given signal to noise ratio (SNR), the potential spatial resolution is then limited by the fluence and the contrast. For instance, at the carbon K-edge, one would expect on the order of only 10 counts/channel for a pixel size of $100 \mathrm{~nm}$ at 10 electrons $/ \AA^{2}$. Depending on the contrast between the carbon K-edges of the materials to be mapped, 10 counts/channel may not be sufficient even for direct electron detectors.

For many polymers, the beam-induced chemistry is reproducible for a given fluence rate and temperature. Particularly at cryogenic temperatures, there is often a significant range of fluences over which chemical changes are apparent before significant mass loss or morphology change, as diffusion of beam-induced products is limited. In some cases, the radiolytic products can even create more contrast than the initial material. However, simply increasing the total fluence yields spectra with a less interpretable, overlapping mix of damaged and undamaged features. Recording a sequence of spectra, each at the minimum fluence set by the detector SNR, creates a two-dimensional fluence-dependent spectrum that contains any contrast from the initial state of the polymers as well as any contrast caused by beam-induced chemistry.

For STEM-EELS mapping, multi-frame averaging has the benefits of lowering the fluence rate for a given total fluence, and allowing drift and distortion correction between frames when there is sufficient concurrent annular dark field (ADF) contrast [1,2]. With direct electron detectors, the noise penalty for splitting the fluence over multiple shorter exposures is reduced [3-6]. On a charge-coupled device (CCD) detector, some care must be taken to sample the sequential fluence spectra across the detector to avoid adding intrinsic noise. For the case of beam-sensitive polymers, a four-dimensional fluence-dependent EELS map can be collected and analyzed using traditional fitting methods. Least squares fitting to references can be easily extended to fluence-dependent spectra, and a variety of readily-available, reference-free machine learning and deconvolution methods used for EELS are already compatible with multidimensional data.

This approach is demonstrated for a model blend of polycarbonate and poly(styrene-acrylonitrile) (PCSAN) on a CCD-style detector. PC-SAN serves as an example that gains or loses contrast in different areas of the component spectra as a result of beam-induced chemistry. The SNR of the resulting fluencedependent linear least squares fit maps improves more rapidly over the initial range of fluences, in which there are relatively dramatic changes to the PC carbon K-edge, than the raw increase in total counts would predict. ADF contrast and $t / \lambda$ are tracked to estimate mass loss and morphology change; changes to the morphology are below the resolution of the map well after radiolytic damage is clear in the spectra. Similar improvements are found for principal component analysis of fluence-dependent spectra. 
Ultimately, the spatial resolution of STEM-EELS for polymers is still limited by fluence. As a matter of practice, it is sensible to always retain the fluence-dependent spectra for references and maps, as there may be unique and identifiable changes to the spectra. For many cases, it is possible to obtain accurate polymer type identification and mapping at pixel sizes that require fluences beyond any assessment of a critical fluence. As EELS detector sensitivity and readout speed continue to increase, retaining the fluence-dependence in hyperspectral mapping should be generically practical for sensitive samples, even those where the changes are not as dramatic as polymers.

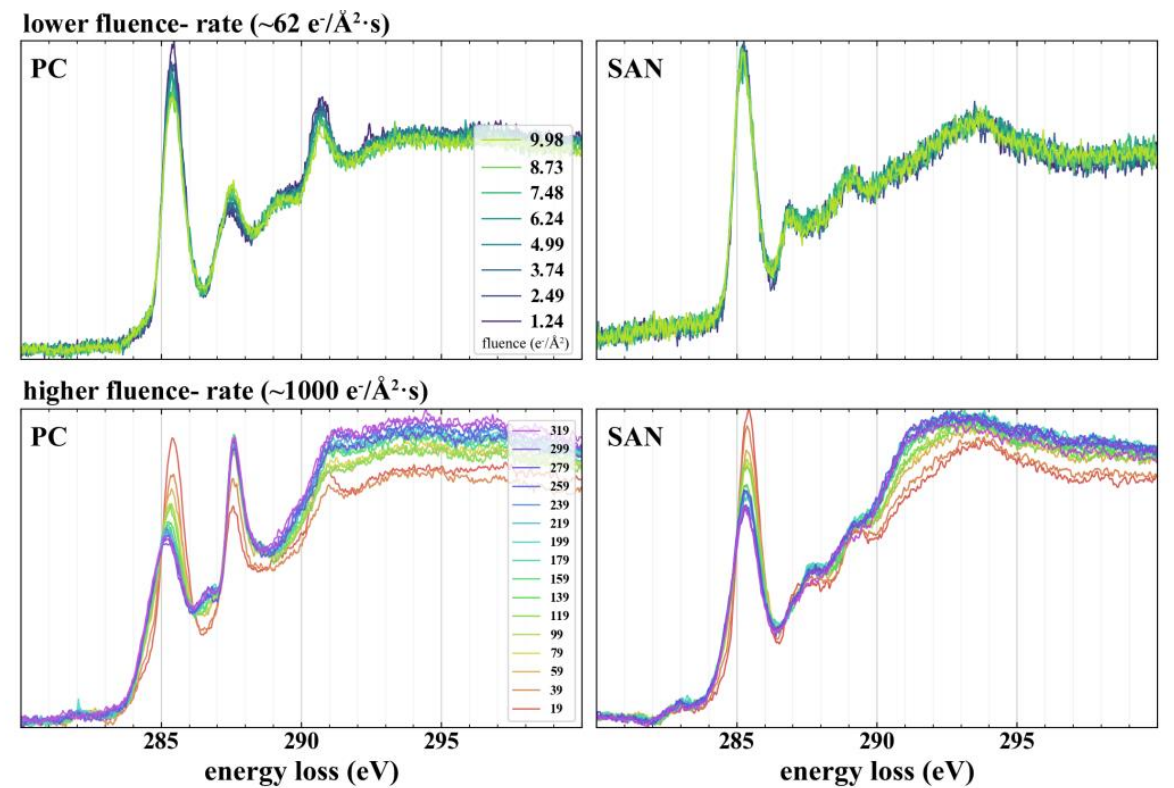

Figure 1. Example fluence-dependent spectra for polycarbonate (PC) and (3:1) poly(styrene-acrylonitrile) (SAN) acquired at $-170^{\circ} \mathrm{C}$ with lower and higher fluence-rates, the latter being appropriate for mapping with $\sim 50$-nm sized pixels using a CCD-based spectrometer. These references are averaged over many pixels across $2-5 \mu \mathrm{m} 2$ areas (the SNR nominally scales with the area under these conditions). For individual spectra, the contrast is not necessarily sufficient to differentiate PC from SAN with good SNR. Even at the low fluence of $\sim 1$ electrons/Å2, PC evidences a decrease in the features commonly attributed to $\mathrm{C}=\mathrm{C}(\sim 285.5 \mathrm{eV})$ and $\mathrm{C}=\mathrm{O} \pi^{*}(\sim 291 \mathrm{eV})$, with a concurrent increase in the feature near $\sim 287.5 \mathrm{eV}$ consistent with $\mathrm{C} \equiv \mathrm{O}$, presumably a beam-induced product. At higher fluences, the dramatic growth of the sharp feature at $\sim 287.5 \mathrm{eV}$ leads to greater contrast than the initial spectra. These fluence-dependent references can be used for identification without the loss of feature fidelity that would result from simply summing the spectra over the same fluence.

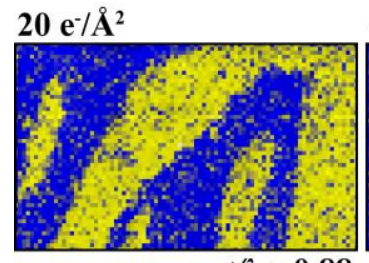

$t / \lambda \approx 0.88$ $40 \mathrm{e}^{-} / \AA^{2}$

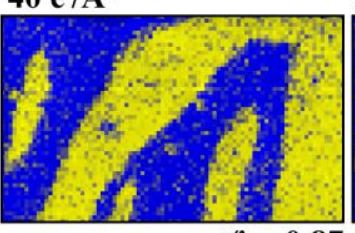

$t / \lambda \approx 0.87$ $80 \mathrm{e} / \AA^{2}$

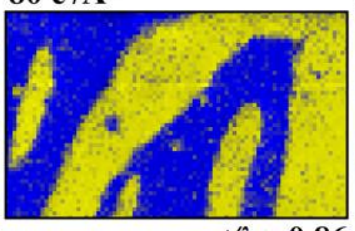

$t / \lambda \approx 0.86$
$160 \mathrm{e}^{-} / \AA^{2}$

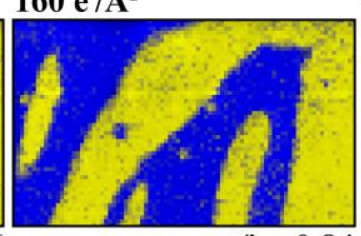

$t / \lambda \approx 0.84$
$320 \mathrm{e} / \AA^{2}$

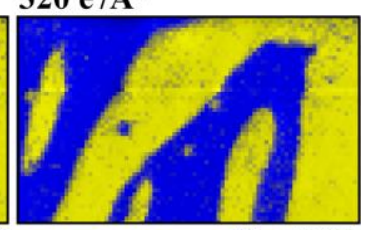

$t / \lambda \approx 0.81$

Figure 2. Linear least squares fits to fluence-dependent references for PC and SAN. Pixel size is $\sim 50 \mathrm{~nm}$, and the lateral field of view is $17.2 \mu \mathrm{m}$. The map was collected with relatively high $\sim 25$ electrons/Å2 per pass on a CCDbased spectrometer. The maps are fits to the data truncated at the various listed total fluences, with the first map being a traditional one-dimensional linear least squares fit. The significant initial improvement to SNR with fluence diminishes after $\sim 100$ electrons/ $\AA 2$ : a better correlation to the change in beam-induced spectral contrast, rather than to the sheer number of electron counts. 


\section{References}

1. Wang, Y, et al. Ultramicroscopy, 84, (2018).

2. Jones, L, et al. Microscopy, 67, (2018).

3. Leitjen, ZJWA, et al. Ultramicroscopy 208, (2020).

4. Maigné, A, et al. Microscopy 67, (2018).

5. Hart, JL, et al. Scientific Reports 7, (2017).

6. Plotkin-Swing, B, et al. Ultramicroscopy 217, (2020). 